TI 2004-068/1

Tinbergen Institute Discussion Paper

\title{
A Note on Costly Sequential Search and Oligopoly Pricing
}

\author{
Maarten C.W. Janssen \\ José Luis Moraga-González* \\ Matthijs R. Wildenbeest
}

Faculty of Economics, Erasmus Universiteit Rotterdam, and Tinbergen Institute.

* CESifo 


\section{Tinbergen Institute}

The Tinbergen Institute is the institute for economic research of the Erasmus Universiteit Rotterdam, Universiteit van Amsterdam, and Vrije Universiteit Amsterdam.

Tinbergen Institute Amsterdam

Roetersstraat 31

1018 WB Amsterdam

The Netherlands

Tel.: $\quad+31(0) 205513500$

Fax: $\quad+31(0) 205513555$

Tinbergen Institute Rotterdam

Burg. Oudlaan 50

3062 PA Rotterdam

The Netherlands

Tel.: $\quad+31(0) 104088900$

Fax: $\quad+31(0) 104089031$

Please send questions and/or remarks of nonscientific nature to driessen@tinbergen.nl.

Most TI discussion papers can be downloaded at http://www.tinbergen.nl. 


\title{
A Note on Costly Sequential Search and Oligopoly PRICING
}

\author{
Maarten C. W. Janssen \\ Erasmus University and Tinbergen Institute \\ José Luis Moraga-González \\ Erasmus University, Tinbergen Institute and CESifo \\ Matthijs R. Wildenbeest \\ Erasmus University and Tinbergen Institute *
}

First version: June 2004

\begin{abstract}
We modify the paper of Stahl (1989) on sequential consumer search in an oligopoly context by relaxing the assumption that consumers obtain the first price quotation for free. When all price quotations are costly to obtain, a new equilibrium arises where consumers randomize between not searching at all and searching for one price. The region of parameters for which this equilibrium exists becomes larger as the number of shoppers decreases and/or the number of firms increases. The comparative statics properties of this new equilibrium are interesting. In particular, the expected price increases as search cost decreases, and is constant in the number of shoppers and in the number of firms. We show that the Diamond result never obtains with truly costly search.
\end{abstract}

Keywords: sequential consumer search, oligopoly, price dispersion

JEL Classification: C13, D40, D83, L13

*Address for correspondence: Erasmus University Rotterdam, Department of Economics, Office H07-26, Postbox 1738, 3000 DR Rotterdam, The Netherlands. Fax: ++31-10-4089149. E-mails: <janssen@few.eur.nl>, $<$ moraga@few.eur.nl> and <wildenbeest@few.eur.nl>. 


\section{Introduction}

One of the most celebrated articles in the consumer search literature is the article by Stahl (1989). Stahl (1989) studies a search model with two types of consumers and where firms price strategically. Fully informed consumers (referred to as shoppers in his article) have no opportunity cost of time and search for all prices at no cost; non-shoppers search sequentially, i.e., they first observe one price and then decide whether or not to observe a second price, and so on. ${ }^{1}$ Stahl (1989) assumes that consumers observe the first price quotation for free, as many other papers in the search literature, ${ }^{2}$ which implies that every buyer makes at least one search. In this paper, we study the implications of relaxing this assumption.

The optimal sequential search rule implies that a consumer continues searching if, and only if, the observed prices are above a certain reservation price. Knowing this, no firm will wish to charge prices above the reservation price. Therefore, under the assumption that obtaining the first price observation is costless, buyers search exactly once in equilibrium and buy at the observed price. In this note, we call this the full participation equilibrium. This equilibrium is one of the two possible equilibrium configurations when the first price quotation is not for free. The new equilibrium that arises is one of partial participation, where some consumers decide not to search at all as they rationally expect prices to be so high that they are indifferent between searching and not searching. The existence and characterization of this new equilibrium is one of the two main contributions of this note.

The other main contribution is to provide a full comparative statics analysis of the effects of all the possible changes in the exogenous parameters. The main comparative statics effects under partial participation are as follows. A first result is that when search costs decrease, expected price increases. This result is mainly due to the fact that an increase in search cost implies more participation of non-shoppers who search only once. As firms have monopoly power over these consumers, they raise their prices. A second result is that an increase in the number of shoppers does not have an effect on expected price as their presence is exactly offset by an increase in the participation of non-shoppers. A last important result is that, unlike in Stahl's model, expected price does not tend to the monopoly price when the number of shoppers converges to zero, nor

\footnotetext{
${ }^{1}$ The implications of sequential consumer search are also examined in, e.g., Anderson and Renault (1999), Reinganum (1979), Rob (1985), Stahl (1996) and Stigtlitz (1987).

${ }^{2}$ An important example in the non-sequential search literature using this assumption is Burdett and Judd (1983). Janssen and Moraga-González (2004) is an exception. This paper studies the implications of entry in a model of non-sequential search.
} 
when the number of firms increases without bound. This is due to the fact that when the fraction of shoppers becomes very small, or when the number of firms increases beyond a critical value, the economy turns into a partial participation equilibrium and in such an equilibrium expected price is insensitive to changes in these parameters.

The rest of this note is organized as follows. Section 2 presents the model. A full characterization and an overview of the two types of equilibria is given in Section 3. Section 4 presents the different comparative statics results and Section 5 concludes.

\section{The Model}

We examine the model of oligopolistic competition and sequential consumer search presented in Stahl (1989), but we assume that all price quotations are costly to acquire for non-shoppers. The features of the model are as follows. There are $N$ firms that produce a homogeneous good at constant returns to scale. Their identical unit cost can be normalized to zero and prices can be interpreted as price-to-cost margins. There is a unit mass of buyers and we assume that buyers hold inelastic demands. ${ }^{3}$ A consumer wishes to purchase at most a single unit of the good and his/her valuation for the item is $v>0$. A proportion $\mu \in(0,1)$ of the consumers has negligible opportunity cost of time and therefore searches for prices costlessly. These consumers are referred to as shoppers. The other $1-\mu$ percent of the buyers, referred to as non-shoppers, must pay search cost $c>0$ to observe every price quotation they get, including the first one. The non-shoppers search sequentially, i.e., a buyer first decides whether to sample a first firm or not and then, upon observation of the price of the first firm, decides to search for a second price or not, and so on. We assume that $v>c$.

Firms and buyers play the following game. An individual firm chooses its price taking price choices of the rivals as well as consumers' search behavior as given. Likewise, an individual buyer forms conjectures about the distribution of prices in the market and decides on his/her optimal search strategy. We restrict the analysis to symmetric Nash equilibria. The distribution of prices charged by a firm is denoted by $F(p)$.

\footnotetext{
${ }^{3}$ Stahl (1989) considers a more general specification of the demand function. The assumption of inelastic demand allows us to compute explicitly the reservation price and give a full characterization of which equilibrium exists for which configurations of parameters. The qualitative results do not depend on this assumption though.
} 


\section{Equilibria}

We first derive some auxiliary results.

Lemma 1 An equilibrium where non-shoppers do not search at all does not exist.

Proof. Suppose non-shoppers did not search. Then the only consumers left in the market would be the shoppers. Therefore, competition between stores would drive prices down to marginal cost. But then, as $v-c>0$, the non-shoppers would gain by deviating and searching once.

Lemma 1 reveals that existence of equilibrium requires the non-shoppers to be active in the market with strictly positive probability. The next result is provided by Stahl (1989).

Lemma 2 In equilibrium non-shoppers will not search beyond the first firm.

Proof. See Lemma 2 of Stahl (1989).

The idea behind Lemma 2 is that pricing above consumers' reservation price is never optimal for firms since buyers will continue searching; as a result, the price buyers will find at the first store they encounter will always be accepted and no further search will take place.

Let us introduce the following notation. Let $\theta_{1}$ be the probability with which a non-shopper searches for a price quotation. Lemmas 1 and 2 together imply that only two candidates for equilibrium exist: either (a) $\theta_{1}=1$, or (b) $0<\theta_{1}<1$. The first case is analyzed in Stahl (1989). We shall refer to this equilibrium as an equilibrium with full consumer participation. This is because if all consumers sample one firm they will all buy. This contrasts with case (b) where consumers mix between not searching at all and searching once so not all consumers will conduct a transaction. Let $\theta_{0}$ denote the probability with which a non-shopper does not search; then an equilibrium with partial consumer participation is characterized by $\theta_{0}+\theta_{1}=1,0<\theta_{1}<1$.

The next remark is that, since $\theta_{1}>0$ in any equilibrium, the equilibrium price distribution must be atomless:

Lemma 3 Irrespective of the search behavior of non-shoppers, if $F(p)$ is an equilibrium price distribution, then it is atomless. Hence, there is no pure strategy equilibrium.

Proof. See Lemma 1 of Stahl (1989). The proof extends straightforwardly to the case of partial consumer participation. 
We note that firms have an incentive to charge low prices in order to attract all the shoppers but at the same time they also have an incentive to charge high prices to extract income from the consumers who do not compare prices. These two forces are balanced when firms randomize their prices. Lemma 3 shows that equilibria must necessarily exhibit price dispersion, and that firm pricing is always characterized by atomless price distributions. In what follows we shall examine the characterization and the existence of the different equilibria.

\section{Case a: Equilibrium with full consumer participation (Stahl, 1989)}

Assume that non-shoppers search for one price with probability 1 , i.e., $\theta_{1}=1$. This is the case analyzed by Stahl (1989) with two modifications. First, as Stahl considers a more general demand structure, an explicit expression for the reservation price cannot be obtained. Second, as Stahl (1989) assumes the first price quotation to be for free, this full participation equilibrium exists for all values of the parameters in his model, but not in ours. We will explicitly define the parameter space for which the full participation equilibrium exists in our case. These two modifications deserve a slightly extended analysis.

Under full participation, the expected payoff to firm $i$ from charging price $p_{i}$ when its rivals choose a random pricing strategy according to the cumulative distribution $F(\cdot)$ is

$$
\pi_{i}\left(p_{i}, F\left(p_{i}\right)\right)=p_{i}\left[\frac{1-\mu}{N}+\mu\left(1-F\left(p_{i}\right)\right)^{N-1}\right]
$$

This profit expression follows from noting that expected demand faced by a firm stems from the two different groups of consumers. Firm $i$ attracts the $\mu$ shoppers when it charges a price that is lower than its rivals' prices, which happens with probability $\left(1-F\left(p_{i}\right)\right)^{N-1}$. The firm also serves the $1-\mu$ non-shoppers whenever they they visit its store, which occurs with probability $1 / N$.

In equilibrium, a firm must be indifferent between charging any price in the support of $F(\cdot)$. Let us denote the upper bound of $F(\cdot)$ by $\bar{p}$. Any price in the support of $F(\cdot)$ must then satisfy $\pi_{i}\left(p_{i}, F(\cdot)\right)=\pi_{i}(\bar{p})$, i.e.,

$$
p_{i}\left[\frac{1-\mu}{N}+\mu\left(1-F\left(p_{i}\right)\right)^{N-1}\right]=\frac{(1-\mu) \bar{p}}{N}
$$

Solving this equation for the price distribution yields

$$
F(p)=1-\left(\frac{(1-\mu)(\bar{p}-p)}{N \mu p}\right)^{\frac{1}{N-1}} .
$$


Since $F$ is a distribution function there must be some $\underline{p}$ for which $F(\underline{p})=0$. Solving for $\underline{p}$ one obtains the lower bound of the price distribution $\underline{p}=(1-\mu) \bar{p} /(\mu N+(1-\mu))$.

The price distribution (3) represents optimal firm pricing. We now turn to discuss optimal consumer behavior. Consider a buyer who has observed a given price $p$. This consumer will continue searching if the expected benefits from continued search exceed the search costs. We can define the reservation price $\rho$ as the price that makes a consumer indifferent between searching once more and accepting the price at hand; this price satisfies:

$$
\int_{\underline{p}}^{\rho}(\rho-p) f(p) d p=c
$$

No firm will charge a price above $\rho$ since this will lead to continued search (Stahl, 1989). As a result the upper bound $\bar{p}=\rho$. We now derive an expression for $\rho$. Integrating by parts in (4) gives

$$
\rho-E[p]-c=0
$$

To calculate $E[p]$ we solve equation (3) for $p$, which gives

$$
p=\frac{\rho}{1+b N(1-F)^{N-1}},
$$

where $b=\mu /(1-\mu)>0$. We note now that $E[p]=\rho-\int_{\underline{p}}^{\rho} F(p) d p$. By changing variables we can write $E[p]=\int_{0}^{1} p d y$. Plugging $p$ from equation (6) gives, after rewriting,

$$
E[p]=\rho \int_{0}^{1} \frac{d y}{1+b N y^{N-1}}
$$

Equation (7) can be plugged into equation (5) to solve for $\rho$ :

$$
\rho=\frac{c}{1-\int_{0}^{1} \frac{d y}{1+b N y^{N-1}}},
$$

We note that the reservation price $\rho$ increases in $c$ and in $N$, decreases in $\mu$ and is insensitive to $v$.

It must be the case that $\rho \leq v$. In addition, non-shoppers must find it profitable to search once, rather than not searching at all, i.e,

$$
v-E[p]-c>0
$$


Inspection of (5) and (9) implies that $\rho<v$. It is useful to rewrite condition (9) as

$$
1-\int_{0}^{1} \frac{d y}{1+b N y^{N-1}}>\frac{c}{v}
$$

This equation gives the set of parameters for which an equilibrium where buyers search once for sure exists. For future reference, let us denote the left-hand-side of equation (10) as $\Phi(1 ; \mu ; N) .{ }^{4}$ We note that $0<\Phi(1 ; \mu ; N)<1$ for all values of the parameters.

Proposition 1 Let $0<\frac{c}{v} \leq \Phi(1 ; \mu, N)$. Then a search equilibrium with full consumer participation exists where buyers search for one price and firms charge prices from the set $[(1-\mu) \rho /(\mu N+(1-$ $\mu), \rho]$ according to the price distribution (3) where $\bar{p}=\rho$ and $\rho$ solves equation (8).

From (5) and (9) and the continuity of the expression of the expected price, it follows that the reservation price $\rho$ converges to $v$ when $c / v$ approaches $\Phi(1 ; \mu, N)$. The question then arises what happens when search costs are high, in particular when $\frac{c}{v}>\Phi(1 ; \mu, N)$ ? In what follows we show that an equilibrium with partial consumer participation arises. This equilibrium is new in the sequential search literature and its properties are interesting.

\section{Case b: Equilibrium with partial consumer participation}

Now assume that non-shoppers randomize between searching for one price quotation and not searching at all, i.e., $\theta_{0}>0, \theta_{0}+\theta_{1}=1$. The expected payoff to firm $i$ is

$$
\pi_{i}\left(p_{i}, F\left(p_{i}\right)\right)=p_{i}\left[\frac{(1-\mu) \theta_{1}}{N}+\mu\left(1-F\left(p_{i}\right)\right)^{N-1}\right] .
$$

The economic interpretation of equation (11) is analogous to that of equation (1), except that there are now $(1-\mu) \theta_{1}$ non-shoppers active, rather than $1-\mu$. A similar analysis as above yields the following equilibrium price distribution:

$$
F(p)=1-\left(\frac{\theta_{1}(1-\mu)(\bar{p}-p)}{N \mu p}\right)^{\frac{1}{N-1}}
$$

with support $[\underline{p}, \bar{p}]$ where $\underline{p}=(1-\mu) \theta_{1} \bar{p} /\left(\mu N+(1-\mu) \theta_{1}\right)$. We now notice that the upper bound is no longer equal to $\rho$, but equal to $v$. To see this, note that non-shoppers should now be indifferent

\footnotetext{
${ }^{4}$ The number 1 in the arguments of $\Phi(\cdot)$ stands for $\theta_{1}=1$.
} 
between searching for one price and not searching all, i.e., it must be the case that

$$
v-E[p]-c=0
$$

It is obvious that conditions (5) and (13) can only hold together if $\bar{p}=v$.

It remains to check that the distribution function specified in equation (12) with support $[p, v]$ is consistent with optimal search behavior of the non-shoppers. As mentioned above, it must be the case that non-shoppers are indifferent between searching and not searching, i.e., $v-E[p]-c=0$. This condition can be rewritten as:

$$
1-\int_{0}^{1} \frac{d y}{1+\frac{1}{\theta_{1}} b N y^{N-1}}=\frac{c}{v} .
$$

For future reference, denote the left-hand-side of equation (14) as $\Phi\left(\theta_{1}, \mu, N\right)$. Inspection of this function reveals that $\Phi(0, \mu, N)=1$ and that $\Phi\left(\theta_{1}, \mu, N\right)$ is monotonically decreasing in $\theta_{1}$ and increasing in $\mu$. It can also be shown that $\Phi\left(\theta_{1}, \mu, N\right)$ decreases in $N .^{5}$ Then:

Proposition 2 Let $\Phi(1, \mu, N)<\frac{c}{v}<1$. Then a search equilibrium with partial consumer participation exists where firms set prices from the set $\left[(1-\mu) \theta_{1} v /\left(\mu N+(1-\mu) \theta_{1}\right), v\right]$ according to the price distribution (12) and consumers randomize between searching for one price with a probability $\theta_{1}$ which solves equation (14) and with the remaining probability they stay out of the market altogether.

\section{Overview of equilibria}

Two equilibria may arise: either consumers search for one price with probability 1, or they mix between searching and not searching. Inspection of the equations above immediately reveals that whether consumers search more or less in equilibrium depends on three critical model parameters: (i) the value of the purchase compared to the search cost $c / v,(i i)$ the number of consumers with negligible opportunity cost of time $\mu$, and (iii) the number of firms $N$. The regions of parameters for which these equilibria exist are represented in Figure 1 (in these graphs we set $N=2$ ). The left graph exhibits a market with many shoppers while the right one illustrates a market with just a few of them. The decreasing curve represents $\Phi\left(\theta_{1} ; \mu, N\right)$ as a function of $\theta_{1}$.

\footnotetext{
${ }^{5}$ The proof of this statement is omitted to save space.
} 


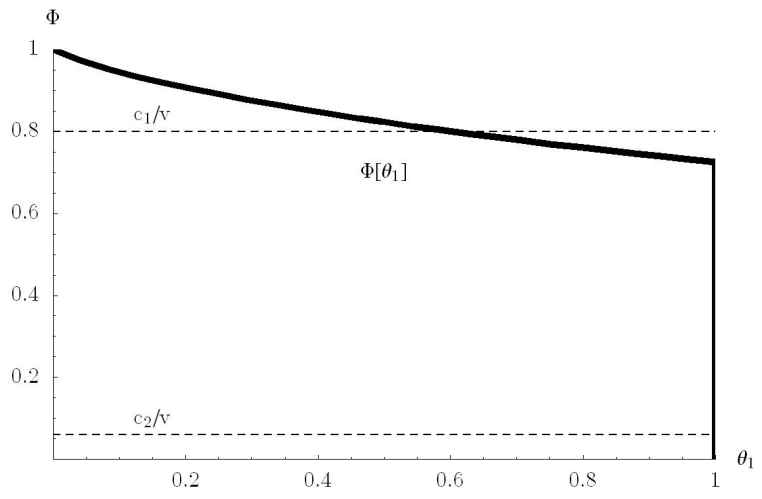

(a) $\mu=0.8$

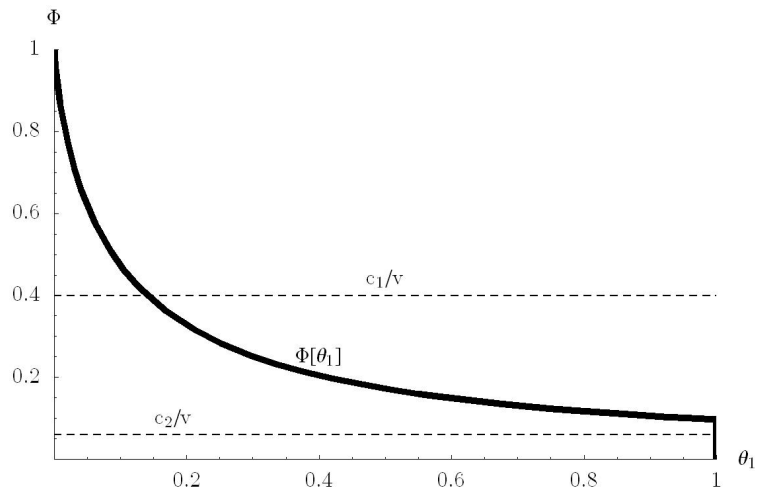

(b) $\mu=0.1$

Figure 1: Equilibrium conditions sequential search $(N=2)$

When there are many shoppers (Figure 1(a)), for large search cost parameters, say $c_{1}$, nonshoppers participate in the market only with probability less than one. This probability is given by the point at which $\Phi(\cdot)$ and $c_{1} / v$, intersect. It can be seen that as search cost falls relative to the value of the purchase, these consumers find it beneficial to search more intensively. When search cost is low enough, e.g. $c_{2}$, non-shoppers search for one price with probability one.

The region of parameters for which an equilibrium with partial consumer participation exists is sensitive to the parameters $\mu$ and $N$. As mentioned above the function $\Phi(\cdot)$ falls as $\mu$ decreases or $N$ increases (see equation (14)). Moreover, when $\mu$ approaches 0 or $N$ becomes very large, $\Phi\left(\theta_{1} ; \mu, N\right)$ approaches 0 for all values of $\theta_{1}$. Figure 1 (b) illustrates a market where $\mu=0.1$. The figure reveals that the existence region of a partial participation equilibrium covers almost the entire parameter space. The same happens when the number of firms grows large. This indicates that the partial participation equilibrium is relevant when the number of firms in the market is large and there are few shoppers.

\section{Comparative Statics}

In this section we study the influence of changes in the parameters of the model on the average price charged in the market. The results are summarized in Table 1. The results for the Stahl (1989) case are known; the others are new. An upwards (downwards) arrow means that the variable under consideration increases (falls); the symbol '-' means that the variable remains constant. In what follows, our discussion shall concentrate on the most striking and interesting observations of the 
equilibrium with partial participation.

\begin{tabular}{|c|c|c|c|c|}
\hline & \multicolumn{2}{|c|}{$\begin{array}{c}\text { Partial consumer } \\
\text { participation }\end{array}$} & \multicolumn{2}{|c|}{$\begin{array}{l}\text { Full consumer parti- } \\
\text { cipation }(\text { Stahl, 1989) }\end{array}$} \\
\hline & $\theta_{1}$ & $E[p]$ & $\rho$ & $E[p]$ \\
\hline$\downarrow c$ & $\uparrow$ & $\uparrow$ & $\downarrow$ & $\downarrow$ \\
\hline$\uparrow \mu$ & $\uparrow$ & - & $\downarrow$ & $\downarrow$ \\
\hline$\uparrow v$ & $\uparrow$ & $\uparrow$ & - & - \\
\hline$\uparrow N$ & $\downarrow$ & - & $\uparrow$ & $\uparrow$ \\
\hline
\end{tabular}

Table 1: Summary of comparative statics results

\section{a. The effects of a reduction in search cost $c$}

The first result we want to emphasize is that a reduction in search cost leads to an increase in the expected price in an equilibrium with partial consumer participation. This follows immediately from the equilibrium condition $v-E[p]-c=0$. To understand the intuition behind this result let us point out that, as Figure 1 shows, the intensity with which non-shoppers search in this type of equilibrium rises as $c$ falls. Note further that these consumers are precisely those who do not exercise price comparisons, and thus they are prepared to accept higher prices. Consequently, a fall in $c$ increases sellers' incentives to charge higher prices more frequently, which in turn raises the expected price.

When $c$ decreases more, the equilibrium will change into one of full participation. Under full participation, a decline in $c$ results in a fall in the reservation price $\rho$ (cf., Stahl (1989)). Inspection of the equilibrium price distribution reveals that $F$ increases as $\rho$ decreases (see Proposition 1). As a result, expected price decreases in $c$.

These observations regarding the influence of a reduction in search cost on expected price-tocost margins are gathered in Figure 2. In Figure 2(a) we have simulated an economy where the number of informed consumers is large $(\mu=0.8)$. This graph depicts expected price as a function of relative search cost and illustrates the comparative statics analysis given above. When there are few shoppers $(\mu=0.1)$ expected price decreases in search cost for almost the entire parameter region (cf., Figure 2(b)). This is because for this parameter constellation the economy is most likely to be in an equilibrium with partial consumer participation.

\section{b. The effects of an increase in the proportion of shoppers $\mu$}

We next consider the effects of an increase in $\mu$. Under partial participation, a change in the number of shoppers does not influence expected price-to-cost margins as nothing changes to the equilibrium 


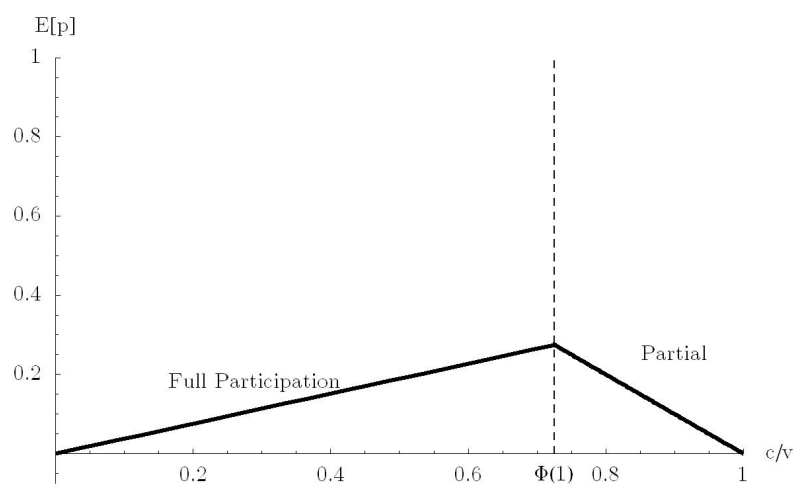

(a) $\mu=0.8$

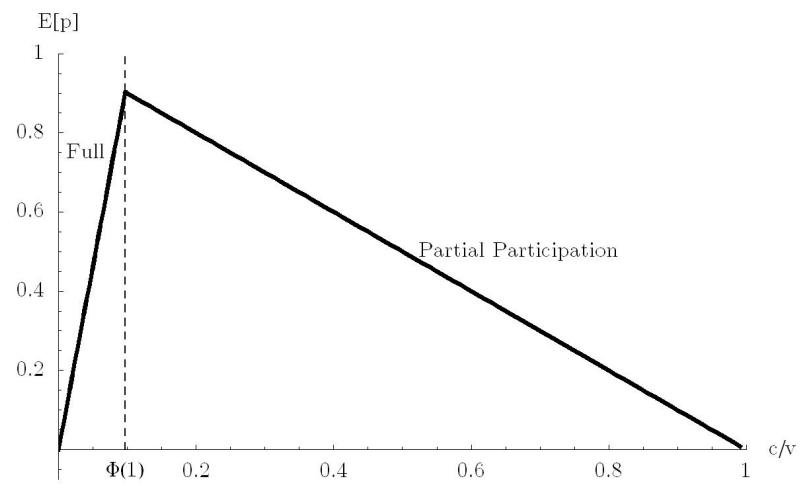

(b) $\mu=0.1$

Figure 2: The influence of lower search $\operatorname{cost} c$ on expected price $(N=2)$

condition $v-E[p]-c=0$. To understand the underlying economic forces of this result, we first note that an increase in $\mu$ has in principle a pro-competitive effect. Keeping the search intensity of non-shoppers constant, firms would tend to charge lower prices with higher probability as there are relatively more shoppers. However, a change in $\mu$ also affects $\theta_{1}$. To see this, one can apply the implicit function theorem to equation (14) to obtain

$$
\frac{d \theta_{1}}{d \mu}=-\frac{\Phi_{\mu}^{\prime}}{\Phi_{\theta_{1}}^{\prime}}=-\frac{\frac{N}{(1-\mu)^{2} \theta_{1}} \int_{0}^{1} \frac{(1-y)^{N-1}}{1+\frac{N \mu(1-y)^{N-1}}{\theta_{1}(1-\mu)}}{ }^{2} d y}{\frac{N \mu}{-(1-\mu) \theta_{1}^{2}} \int_{0}^{1} \frac{{(1-y)^{N-1}}_{1+\frac{N \mu(1-y)^{N-1}}{\theta_{1}(1-\mu)}}^{2}}{1-\mu}}=\frac{\theta_{1}}{\mu(1-\mu)}>0
$$

which means that an increase in $\mu$ results in an increase in the search intensity of the non-shoppers $\theta_{1}$. This is obviously due to the fact that more informed consumers in the market makes searching more attractive for the non-shoppers, as the former buyers put pressure on firms to reduce prices. A higher search intensity of non-shoppers in turn gives firms incentives to increase prices, since non-shoppers do not compare prices. Interestingly, these two opposite forces offset each other so that expected price remains constant.

If non-shoppers search for one price for sure, the pro-competitive effects of an increase in $\mu$ mentioned above are strengthened by the fact that the reservation price $\rho$ decreases in $\mu$ and thus expected prices fall (cf., Stahl (1989)). These remarks are gathered and illustrated in Figure 3.

Figure 3(a) simulates an economy where product's valuation is relatively low compared to search $\operatorname{cost}(c / v=0.5)$. The figure depicts expected price-to-cost margins as a function of $\mu$. As $\Phi(1 ; \mu ; N)$ is decreasing in $\mu$, it easily follows that for a given $N$ and $c / v$, there is a unique $\widehat{\mu}$ such that inequality 


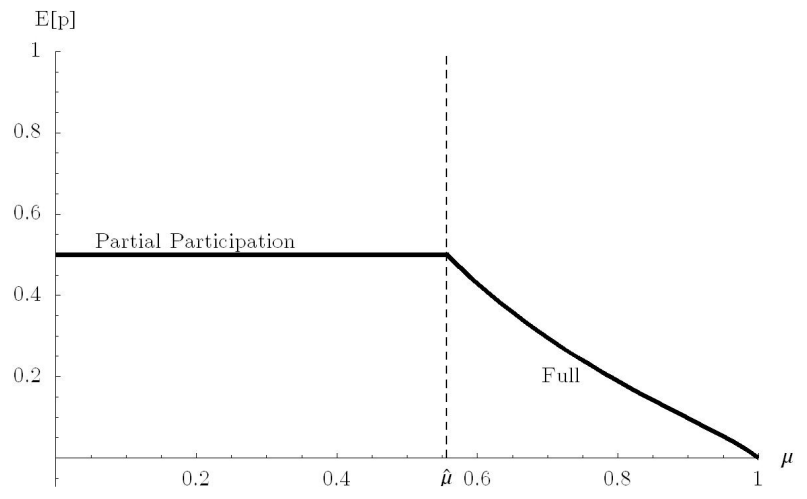

(a) $c / v=0.5$

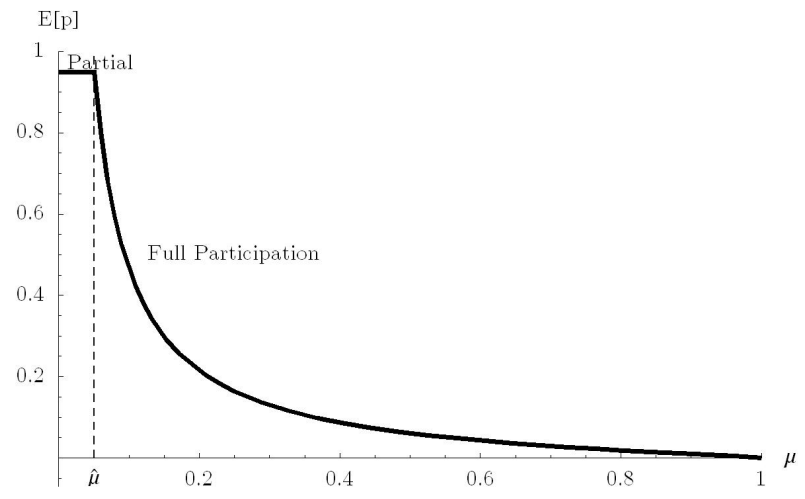

(b) $c / v=0.05$

Figure 3: The influence of $\mu$ on expected price $(N=2)$.

(10) holds with equality. Therefore, the market equilibrium exhibits partial participation when $\mu$ lies in the interval $(0, \widehat{\mu})$ and it exhibits full participation when $\mu$ lies in the interval $(\widehat{\mu}, 1)$. It is straightforward to see that, starting from an equilibrium with full consumer participation, as $\mu \rightarrow 0$ the economy eventually moves into an equilibrium with only partial participation so the Diamond result cannot arise in our model. In Figure 3(b) we have simulated an economy where search cost is relatively low $(c / v=0.05)$. The only difference is that the region of parameters for which consumers search for one price with probability 1 is much larger than before.

\section{c. The effects of an increase in the value of the purchase $v$}

We next briefly focus on changes in $v$. For convenience we shall graph expected price against $c / v$. The main difference with the (reverse) effects of a change in $c$, is that under full participation $c$ affects $\rho$, whereas $v$ does not affect $\rho$. So, the only difference with case a. is that now when buyers search for one price for sure, an increase in $v$ does not alter the reservation price of the non-shoppers $\rho$ so expected margins remain unchanged.

The graphs of Figure 4 show the influence of an increase in $v$ on expected prices. Figure 4(a) simulates an economy where search costs and the number of informed consumers are relatively high ( $c=0.5$ and $\mu=0.8)$. When $c / v$ lies in the interval $(\Phi(1), 1)$, non-shoppers do not participate in the market surely. In this parameter area, the expected margin rises as $v$ increases. When $c / v$ is between 0 and $\Phi(1)$, non-shoppers search for one price with probability 1. For this region of parameters expected margins are unaffected by a change in $v$. Figure 4(b) shows the case of an economy with relatively few shoppers. 


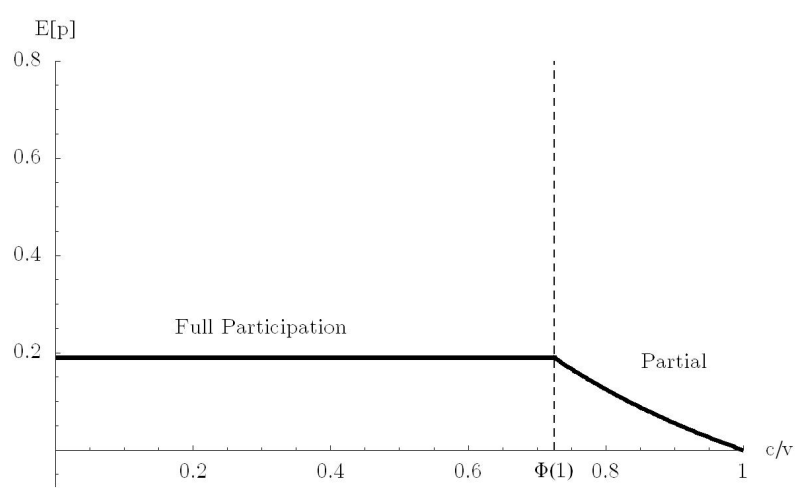

(a) $c=0.5$ and $\mu=0.8$

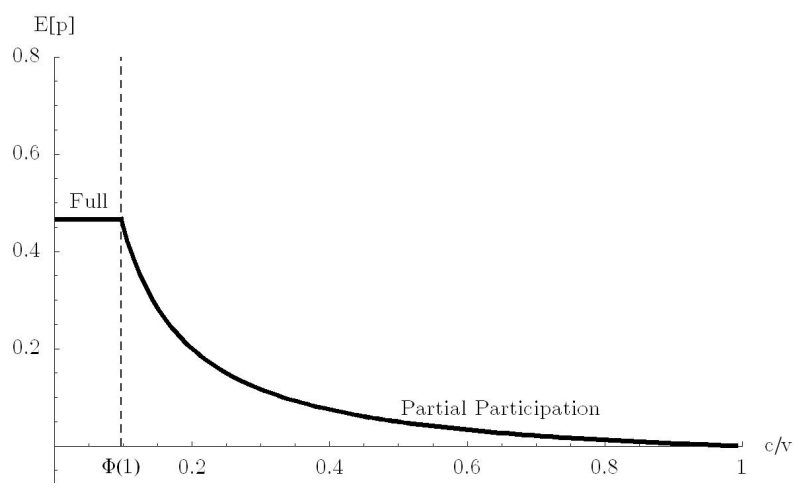

(b) $c=0.05$ and $\mu=0.1$

Figure 4: The impact of $v$ on expected price $(N=2)$

\section{d. The effects of an increase in the number of firms $N$}

Under partial participation, it immediately follows from the equilibrium condition $v-E[p]-c=0$ that an increase in $N$ does not affect expected price. The underlying economic forces that bring about this result are, however, less straightforward. We have noted before that $\Phi\left(\theta_{1} ; \mu ; N\right)$ is decreasing in $N$. This means that without a change in $\theta_{1}$ expected prices would rise: as $N$ increases it becomes more and more unlikely that an individual firm sells to the shoppers and each firm tends to concentrate more and more on selling to the non-shoppers. Therefore, expected price would rise for a given $\theta_{1}$. Higher expected prices means, however, that a larger fraction of non-shoppers prefers not to buy and this effect exactly offsets the first effect.

The analysis for the case of full participation follows Stahl (1989) and he shows that expected prices rise in $N$. Intuitively, only the first effect discussed above is relevant here as $\theta_{1}$ is fixed to be equal to 1 . In the limit, Stahl (1989) shows that expected price converges to the monopoly price and the Diamond result holds.

It is then interesting to see which equilibrium arises for which values of $N$. We will argue that for given $c / v$ and $\mu$ when $N$ becomes sufficiently large, the equilibrium is characterized by partial participation. What happens as $N$ increases is that, if the non-shoppers keep searching for one price with probability one, expected price tend to the monopoly price and eventually, the condition $v-E[p]-c>0$ is violated. This is easily seen upon inspection of $(10)$ and noting that $\Phi(1 ; \mu, N)$ declines monotonically with $N$ and converges to zero as $N$ approaches infinity. The implication of this is that starting from an equilibrium with full participation our model does not yield the 
Diamond result in the limit since at some point the economy turns to a partial participation equilibrium. Figure 5 below illustrates the comparative statics results discussed above.

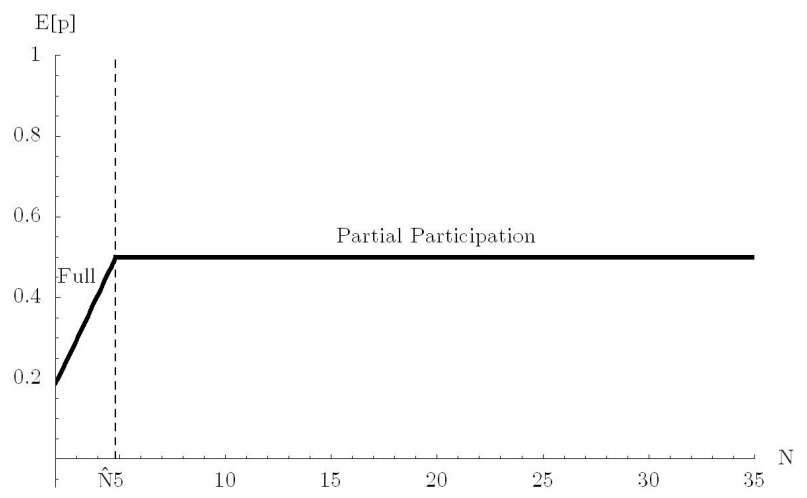

(a) $c=0.5$ and $\mu=0.8$

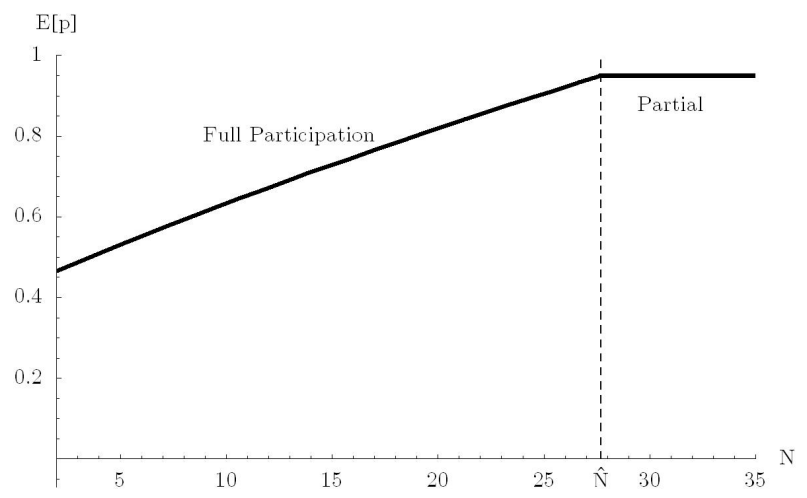

(b) $c=0.05$ and $\mu=0.1$

Figure 5: The impact of $N$ on expected price

\section{Conclusion}

In this note, we have taken the seminal model of Stahl (1989) on sequential consumer search and oligopolistic pricing and studied the implications of relaxing the assumption that consumers obtain the first price quotation for free. When also the first price quotation is obtained at a positive search cost, a new type of equilibrium arises where consumers randomize between not searching and searching for one price, i.e., where there is less than full consumer participation. The partial participation equilibrium exists when search costs are above a certain threshold (depending on the other parameter values). This threshold can be made arbitrarily low provided that the number of firms is large enough and/or the number of shoppers is sufficiently small. Therefore, especially in markets with many firms and/or with few shoppers, this partial participation equilibrium should be seriously considered. This new equilibrium exhibits interesting comparative statics properties. In particular, the expected price increases as search cost decreases, and is constant in the number of shoppers and in the number of firms. Finally, the paper shows that, starting from an equilibrium with full participation, a Diamond result never obtains when the number of shoppers goes to zero and/or the number of firms goes to infinity because with truly costly search the economy eventually moves to an equilibrium with partial consumer participation. 


\section{References}

[1] Simon Anderson and Regis Renault: "Pricing, Product Diversity and Search Costs: A BertrandChamberlin-Diamond Model," Rand Journal of Economics 30-4, 719-735, 1999.

[2] Kenneth Burdett and Kenneth L. Judd: "Equilibrium Price Dispersion," Econometrica 51-4, 955-69, 1983.

[3] Maarten C.W. Janssen and José Luis Moraga-González: "Strategic Pricing, Consumer Search and the Number of Firms," Review of Economic Studies (forthcoming).

[4] Jennifer F. Reinganum: "A Simple Model of Equilibrium Price Dispersion," Journal of Political Economy 87, 851-58, 1979.

[5] Raphael Rob: "Equilibrium price distributions," Review of Economic Studies 52, 457-504, 1985.

[6] Dale O. Stahl: "Oligopolistic Pricing with Sequential Consumer Search," American Economic Review 79, 700-12, 1989.

[7] Dale O. Stahl: "Oligopolistic Pricing with Heterogeneous Consumer Search," International Journal of Industrial Organization 14, 243-68, 1996.

[8] Joseph Stiglitz: "Competition and the number of firms in a market: Are duopolies more competitive than atomistic markets?" Journal of Political Economy 95, 1041-1061, 1987. 